Supporting Information

\title{
Adhesion Strengthening Mechanism of Carbon Nanotube- Embedded Epoxy Composites: A Fracture-Based Approach
}

Shuhuan $\mathrm{Hu}^{\text {abcd}}$, Wei Huang ${ }^{\mathrm{b} \S}$, Fanchao Meng ${ }^{\mathrm{cd}}$, Raymond H. W. Lam ${ }^{\mathrm{be}}$ and Denvid Lau ${ }^{\mathrm{a} \mathrm{e}^{*}}$

a Department of Architecture and Civil Engineering, City University of Hong Kong, Hong Kong, 999077, China

${ }^{\mathrm{b}}$ Department of Biomedical Engineering, City University of Hong Kong, Hong Kong, 999077, China

${ }^{\mathrm{c}}$ BGI-Shenzhen, Shenzhen, Guangdong, 518083, China

${ }^{d}$ Guangdong High-throughput Sequencing Research Center, Guangdong, 518000, China

e City University of Hong Kong Shenzhen Research Institute, Shenzhen, Guangdong, 518057, China

$\S$ These authors contributed equally to this work.

* Correspondence and requests for materials should be addressed to Denvid Lau (email: denvid.lau@cityu.edu.hk) and Raymond H. W. Lam (email: rhwlam@cityu.edu.hk). 


\section{Supplemental Figures}

1. Sputter Gold on Silicon

Gold

Silicon

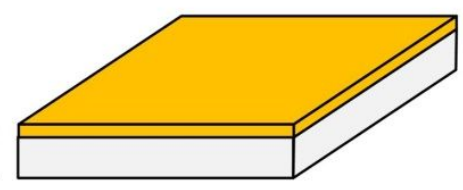

2. Pattern Gold by Photolithography

Gold

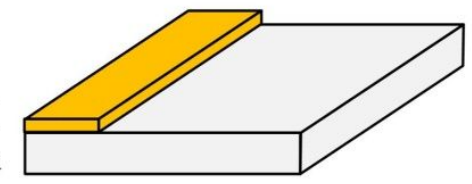

3. Spincoat and Pattern SU-8/CNT

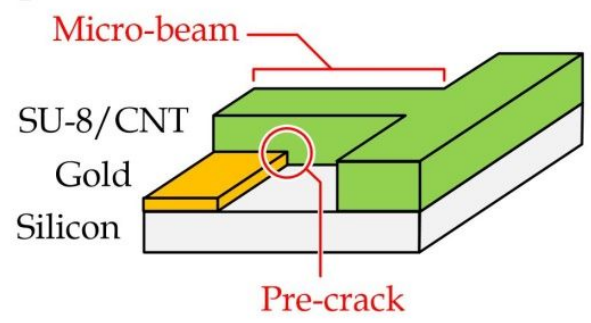

4. Sputter Chromium (Multiple Runs)

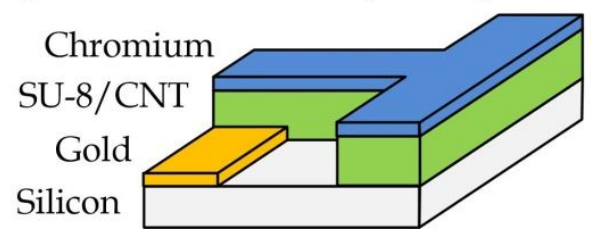

Figure S1: Key fabrication steps. An SU-8/CNT composite micro-beam is generated on silicon, sandwiching a patterned gold layer as the pre-crack. An overhead chromium layer is then deposited for inducing stress in the layers and debonding the micro-beam. 

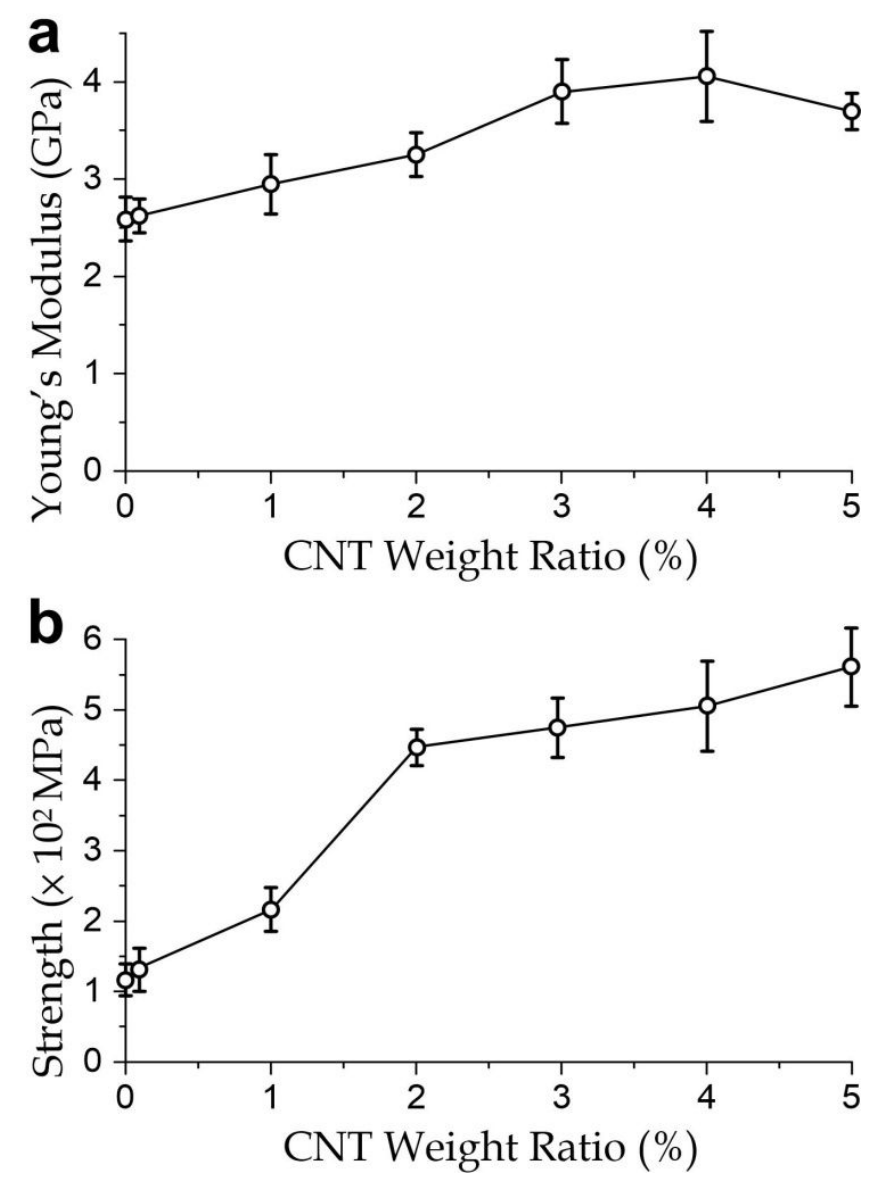

Figure S2: Mechanical properties of SU-8/CNT composites. (a) Young's modulus and (b) strength of the SU-8/CNT composite for different CNT weight ratios ranging $0 \%-5 \%$. Error bars are standard deviations. 

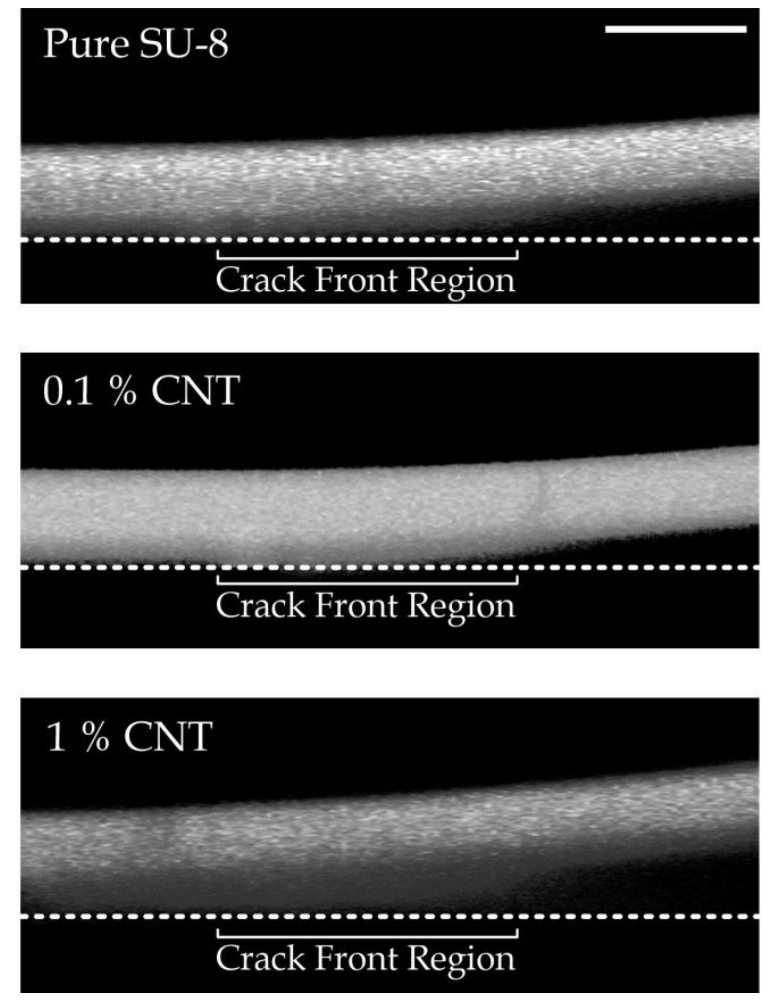

Figure S3: Confocal fluorescence micrographs of debonded SU-8/CNT micro-beams. Crack front regions of the debonded SU-8/CNT micro-beams with different CNT weight ratios (0\%, $0.1 \%$ and $1 \%)$ are provided here. SU-8 is visualized with its own fluorescence properties. Distribution of CNTs can be identified as the darker/black spots within a bulk SU-8 body. Scale bar: $30 \mu \mathrm{m}$. 

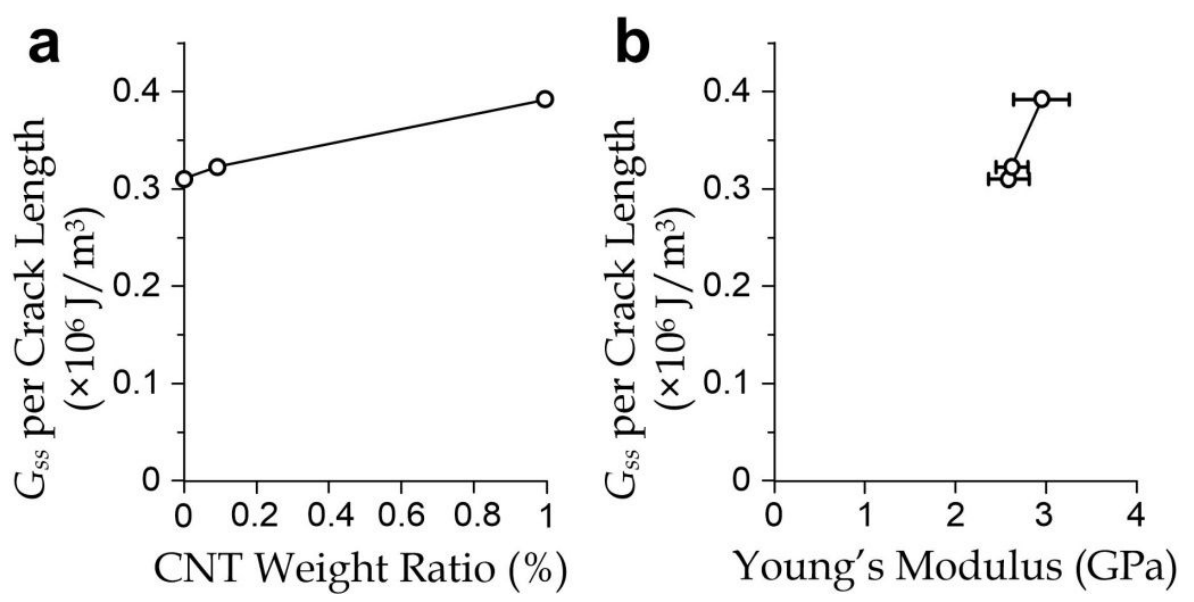

Figure S4: Energy release rate $\left(G_{s s}\right)$ per induced crack length. (a) $G_{S S}$ per crack length versus CNT weight ratio. (b) $G_{s s}$ per crack length versus Young's modulus of the SU-8/CNT composite. Error bars are standard deviations. 


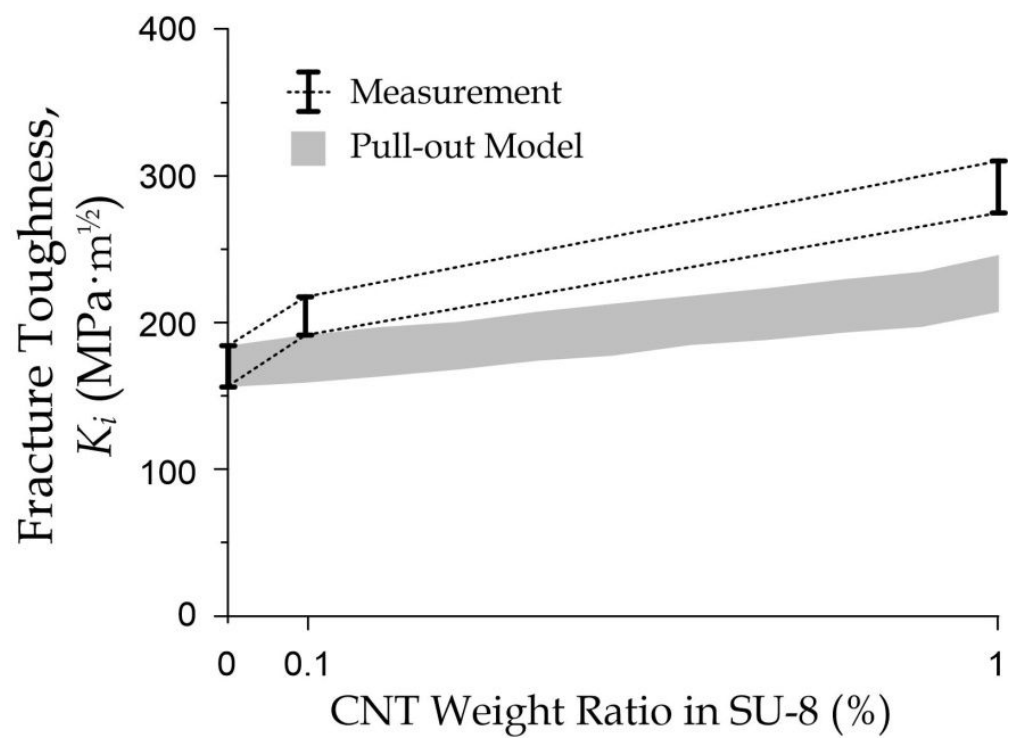

Figure S5: Enhancement of the interfacial fracture energy based on the pull-out toughening mechanisms for different weight ratios of CNT in SU-8. 
a

\section{SU-8 Photoresist}

1. Sputter and Pattern Gold on Substrate

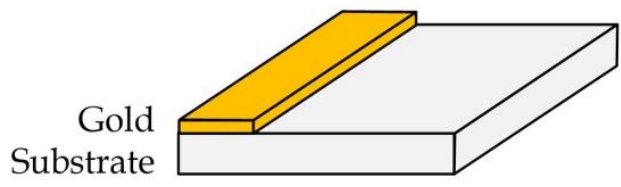

2. Deposit Polymer Composite

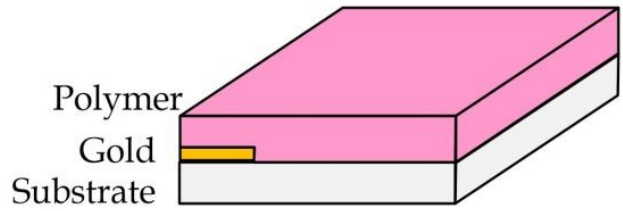

3. Spincoat and Pattern SU-8

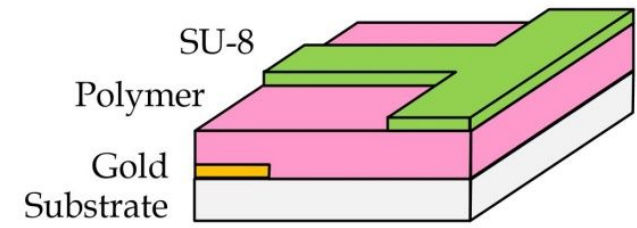

4. Etch Polymer and Remove SU-8

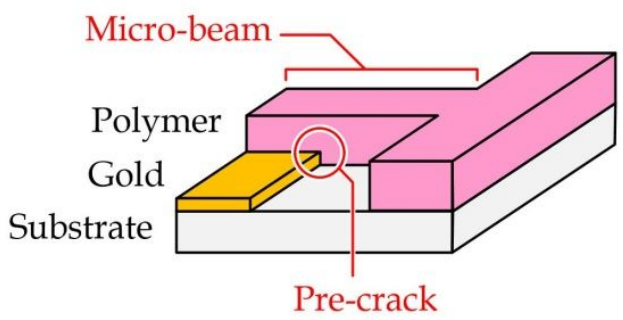

b

\section{SU-8 Molding}

1. Sputter and Pattern Gold on Substrate Substrate

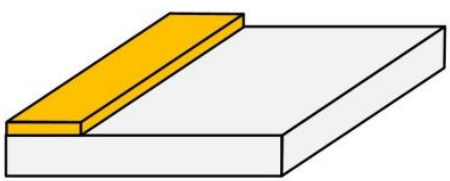

2. Spincoat and Pattern SU-8 Mold

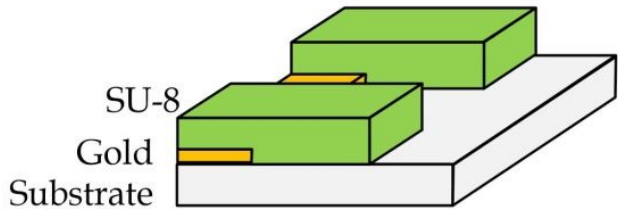

3. Fill Polymer Composite in Mold Cavity

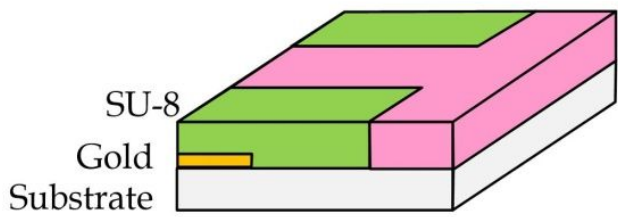

4. Remove SU-8

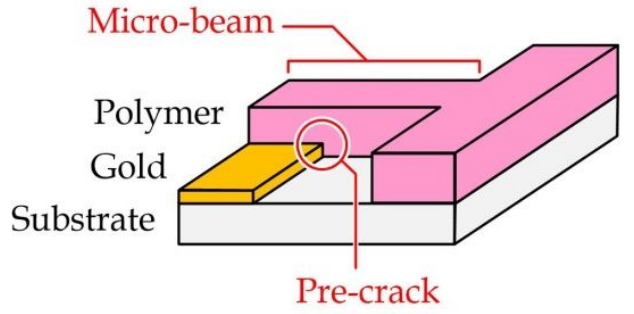

Figure S6: Alternative microfabrication approaches for non-photo-patternable

polymer/particulate composites. (a) SU-8 can function as photoresist for photolithography of polymer composites, whose etchant is also available. (b) SU-8 can be patterned as a mold for polymer composites. After curing of the polymer, the SU-8 mold is removed chemically (Remover PG, Microchem, MA, USA). 
